\title{
Spike in neuroimaging requests following the conviction of the optometrist Honey Rose
}

Eye (2018) 32, 489-490; doi:10.1038/eye.2017.274; published online 8 December 2017

The conviction of the optometrist Honey Rose for manslaughter after a missed diagnosis of papilloedema in 8-year-old Vincent Barker was recently quashed by the court of appeal. ${ }^{1}$ The case has led to an increased awareness of disc pathology and a rise in referrals from community optometrists. We sought to explore whether or not this had led to an increase in neuroimaging requests.

We used our Trust's radiology patient data management system to extract anonymised information from the 92978 CT and MRI head requests made over a 3-year period from May 2014. For each scan, we performed automated text searches for the word 'papilloedema' and related terms, including spelling and phrase variations. We searched the clinical information provided by the referring clinician as well as the final radiology report.

We confined our analysis to patients without previous imaging in the study period or a month prior. Statistical analysis was performed using Stata v14 (Stata Corp, College Station, TX, USA).

We found that scan requests referencing papilloedema increased from 13.6 per month $(0.85 \%)$ pre-August 2016 to $15.9(1.1 \%)$ thereafter $(P=0.02)$. This trend was most visible in A\&E where it was usual for one or two scans to be requested in most months (Figure 1). Following the trial in August 2016, there was a spike in referrals with six requests in October and an overall increase from 0.9 to 2.1 requests per month $(P=0.01)$ following August 2016. A similar but less dramatic effect was seen for requests originating from ophthalmology, which increased from 5.5 to 6.75 requests per month $(P=0.04)$.
The increase we observed is likely related to the publicity surrounding the court case, based on the close temporal relationship between the conviction and the spike in imaging referrals. Optometrist have previously been prosecuted in the civil courts for missed diagnoses of papilloedema, ${ }^{2}$ but this is the first time in the United Kingdom that criminal charges have been brought. Ironically, it was the failure to make the correct diagnosis that led to the conviction being overturned, as the consequences of the miss were not deemed to be 'foreseeable'. The primary breach of duty was then judged a matter for the regulator. ${ }^{1}$

With an increasingly team-based approach in optometry and ophthalmology, the challenge for courts and regulators will be in balancing the failures of the individual against those of systems and institutions in order to best avoid the damaging effects of a shift to defensive decision-making and a rise in investigationrelated morbidity. ${ }^{3}$

The use of imaging, including fundus photography, OCT, FAF, FFA and B-scan ultrasonography can be helpful in distinguishing papilloedema from pseudopapilloedema, and, along with specialised expertise, may account for the attenuated rise in requests originating from ophthalmology. However, these modalities have their own limitations, ${ }^{4}$ and robust IT systems are required to ensure that, where requested, these investigations are appropriately reviewed and acted upon.

Contemporaneous fundus photographs of Vincent Barker showing obvious bilateral disc swelling were available at the trial, but there was some question over whether or not they had been seen on the day he was examined. ${ }^{5}$

\section{Conflict of interest}

The authors declare no conflict of interest. 


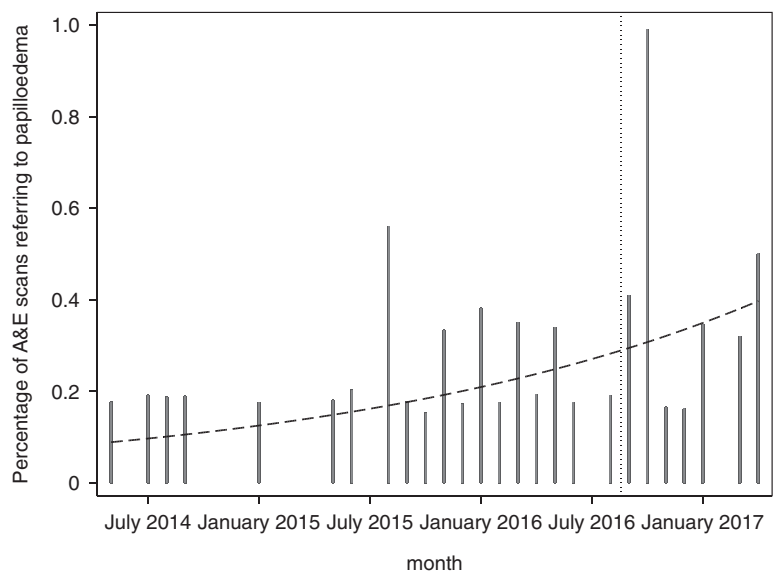

Figure 1 Bar chart showing the proportion of referrals from A\&E referencing papilloedema over time. The dashed line represents postestimation probabilities from a logistic regression model that showed a significant increase $(P=0.005)$ over the study period. The dotted line indicates the date of the original trial.

\section{References}

1 Rawlinson K. Optometrist wins appeal against conviction for manslaughter of boy, 8. The Guardian 2017.

2 Woodward EG. Clinical negligence. Ophthalmic Physiol Opt 2006; 26: 215-216.

3 Pearce MS, Salotti JA, Little MP, McHugh K, Lee C, Kim KP et al. Radiation exposure from CT scans in childhood and subsequent risk of leukaemia and brain tumours: a retrospective cohort study. Lancet 2012; 380: 499-505.

4 Chang MY, Velez FG, Demer JL, Bonelli L, Quiros PA, Arnold AC et al. Accuracy of diagnostic imaging modalities for classifying pediatric eyes as papilledema versus pseudopapilledema. Ophthalmology 2017; 124: 1839-1848.

5 Criminal trial of optom. Optometry Today. Available at: https://www.aop.org.uk/ot/professional-support/clinicaland-regulatory/criminal-trial-of-optometrist-begins. (accessed on 13 August 2017).

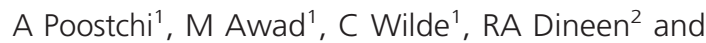

$$
\begin{aligned}
& \text { AM Gruener }{ }^{1,3}
\end{aligned}
$$

${ }^{1}$ Department of Ophthalmology, Queen's Medical Centre, Nottingham University Hospitals NHS Trust, Nottingham, UK

${ }^{2}$ Department of Neuroradiology, Queen's Medical Centre, Nottingham University Hospitals NHS Trust, Nottingham, UK

${ }^{3}$ Division of Neuro-Ophthalmology, Wilmer Eye Institute, Baltimore, MD, USA

Correspondence: AM Gruener, Department of Ophthalmology, Queen's Medical Centre, Nottingham University Hospitals NHS Trust, Nottingham NG7 2UH, UK Tel: +44 115 9249924; Fax: +44 1159709749 .

E-mail: anna.gruener@nuh.nhs.uk 